\title{
Realidad de la formación gerencial en las pequeñas y medianas empresas venezolanas
}

\author{
Hernández, René* \\ Silvestri, Karin** \\ Añez, Silenis*** \\ Gamboa, Luisa****
}

\begin{abstract}
Resumen
El requerimiento de las pequeñas y medianas empresas en alcanzar mayores niveles de productividad y rentabilidad, ameritan la puesta en práctica de políticas y estrategias centradas en el individuo, específicamente en quien dirige la organización. La investigación presentada a continuación tiene como objetivo determinar la realidad de la formación gerencial de los directivos de las pequeñas y medianas empresas (PyME) venezolanas. El estudio se encuentra enmarcado dentro de un diseño no experimental, puesto que su fin es la observación de las variables y no la manipulación de éstas, de la misma manera, este estudio tiene características de diseño transversal o transeccional, ya que se estudia y se describen los datos obtenidos en un período de tiempo determinado, por otro lado la investigación se enmarca dentro de la tipología de campo. Entre las conclusiones del estudio como parte de los objetivos específicos trazados se alcanzo a diseñar el perfil de competencias gerenciales de los directivos de las PyME venezolanas el cual esta conformado por una serie de indicadores como conocimientos, habilidades, rasgos y capacidad de adecuación entre otros.
\end{abstract}

Palabras clave: Formación gerencial, competencias, formación profesional, pequeñas y medianas empresas.

Recibido: 20-03-07. Aceptado: 02-11-07

* Docente-Investigadora de la Universidad del Zulia (LUZ). Miembro del Grupo de Investigación Desarrollo Gerencial. Investigadora acreditada en el Programa de Promoción al Investigador (PPI). E-mail: reneeher@gmail.com

** Profesora Invitada de la Universidad del Zulia en la Facultad de Ciencias Económicas y Sociales. Miembro del Grupo de Investigación Desarrollo Gerencial. Investigadora acreditada en el Programa de promoción al Investigador (PPI).

*** Docente-Investigadora de la Universidad del Zulia en la Facultad de Ciencias Económicas y Sociales (FCES). Investigadora acreditada en el Programa de promoción al Investigador (PPI).

**** Docente-Investigadora, profesora asociada de LUZ-FCES. E-mail: luisagamboa@cant.net 
Realidad de la formación gerencial en las pequeñas y medianas empresas

Hernández, René; Silvestri, Karin; Añez, Silenis y Gamboa, Luisa

\title{
The Reality of Managerial Training in Small and Medium Venezuelan Companies
}

\begin{abstract}
The requirement of small and medium-sized companies to reach greater levels of productivity and profitability, merits putting into practice policies and strategies centered on the individual, specifically the one who directs the organization. The objective of this study was to determine the reality of managerial training for directors of small and medium Venezuelan enterprises (SAMEs PyMEs). The study is framed within a non-experimental design, since its purpose is to observe the variables and not to manipulate them; in the same way, this study has characteristics of a transversal or transectional design, since the data obtained over a certain time period are studied and described; on the other hand the research is framed within the field typology. Among the conclusions of the study as part of the specific objectives laid out, a profile of managerial competences was designed for directors of Venezuelan SAMEs (PyMEs), made up of a series of indicators such as knowledge, abilities, features and adaptation capacity, among others.
\end{abstract}

Key words: Managerial training, competences, professional training, Small and medium enterprises.

\section{Introducción}

El requerimiento de hacer cada día más competitivas a las organizaciones, lleva a trazarse nuevas estrategias a los individuos que dirigen el destino de las mismas, más aun cuando se trata de gerenciar empresas. La permeabilidad de las pequeñas y medianas empresas ante las turbulencias del entorno genera una continua búsqueda de nuevas directrices y perfeccionamiento de las competencias de los directivos, que permitan disminuir el impacto de los factores sociales, económicos, políticos y tecnológicos.

La búsqueda de optimizar la capacidad gerencial ha llevado a las organizaciones a implementar diferentes modelos gerenciales, entre ellos pueden citarse el outsourcing o subcontratación de terceros, el benchmarking o emulación estratégica, el control de la calidad en sus diversas formas; pero realmente no se ha planteado realizar una revisión de la realidad en las organizaciones venezolanas, según Armas (1999) para que en torno a la particularidad de cada una de ellas y a esa necesidad de gerenciar el conocimiento se ponga en práctica una estrategia acorde con sus propios requerimientos.

Se evidencia entonces el requerimiento de establecer cual es la realidad existente, para estructurar hacia donde debe abocarse la formación gerencial, específicamente en el caso de las PyME, requiriéndose un diagnóstico de la gerencia para sistematizar estrategias capaces de proporcionar fortaleza a la misma, en aquellas áreas en las cuales presente mayores debilidades.

Al referirse a las organizaciones más exitosas de los próximos años, se indica la necesidad de desarrollar la capacidad de liderazgo en la formación gerencial tanto como los otros recursos opera- 
cionales con los que cuenta (Villegas, 1999). Esto va a permitir generar o diseñar un perfil determinado, el cual debe estar conformado por una serie de indicadores que servirán de referencia al momento de establecer comparaciones.

Se plantea además la necesidad del manejo de habilidades elementales de la computadora, comprensión de la tecnología, sus dimensiones, sus características, en fin sus competencias. Todo ello sin perder de perspectiva la realidad en la cual están inmersas las organizaciones (Drucker, 2000), de allí la importancia de realizar investigaciones en el área de la formación gerencial de los directivos de las organizaciones.

Lo señalado en el párrafo anterior refiere también a la sociedad ilustrada en la cual los gerentes deben estar preparados para formarse continuamente, es decir hace alusión a la formación gerencial; en aquellas áreas en las cuales se incorporen nuevos cambios, para hacer más competitivos a los directivos. Esta adopción de nuevas formas de hacer las cosas, involucra la necesidad de conocer cuales son sus necesidades y en que áreas debe actualizarse el gerente.

Se plantean según lo analizado en el problema en consecuencia, las siguientes interrogantes:

¿Cuál es la realidad de la formación gerencial de las PyME en cuanto a las competencias gerenciales de sus directivos?

¿Cuál es la formación profesional de los directivos de las PyME?

En este marco se plantea el objetivo general de la investigación, el cual contempla determinar la realidad de la formación gerencial de los directivos de las pequeñas y medianas empresas (PyME) venezolanas, derivándose así los objetivos específicos los cuales son:

- Establecer el perfil de competencias gerenciales de los directivos de las PyME venezolanas.

- Determinar la formación profesional de los gerentes de las PyME.

La investigación se centra en las pequeñas y medianas empresas (PyME) las cuales son la base fundamental de una economía sólida y para ello hay que buscar estrategias gerenciales y de apoyo que se correspondan con su realidad (Palacios, 1999), entre otras razones ya que el entorno sumamente cambiante, amerita una capacidad de respuesta fundamentada en una gerencia preparada para asumir las oportunidades y amenazas latentes. La investigación es de tipo descriptiva, ya que se pone en práctica para obtener información de un determinado segmento, en este caso el empresariado con la finalidad de procesar dicha información y arrojar resultados orientados a proporcionar un determinado uso (Hernández y col., 2000).

Esta data que arrojaron las unidades de información permitirán el establecimiento de estrategias de formación acordes con las exigencias y demandas de los directivos de las pequeñas y medianas empresas, considerando las expectativas detectadas previamente. denotándose así en una investigación de campo, el cual se define como el análisis sistemático de problemas con el propósito de describirlos, explicar sus causas y efectos, entender su naturaleza y factores constituyentes o predecir su ocurrencia. 
Realidad de la formación gerencial en las pequeñas y medianas empresas Hernández, René; Silvestri, Karin; Añez, Silenis y Gamboa, Luisa

Atendiendo a los parámetros del tipo de diseño la presente investigación se presenta como de tipo no experimental, definida como aquella que se realiza sin manipular deliberadamente las variables (Hernández y col., 2000). Es decir los fenómenos se analizarán tal cual están en el ambiente, en la realidad de las pequeñas y medianas empresas, a ser consultadas a través de las respectivas unidades de información.

La investigación a ser desarrollada requirió del compromiso de las unidades de investigación, el muestreo seleccionado de acuerdo a las exigencias es el probabilístico, de sujetos voluntarios, considerando para ello que se trata de muestras fortuitas (Hernández y col., 2000). Atendiendo a los requerimientos de información y a los objetivos planteados para la investigación, se seleccionó el cuestionario como modalidad de la encuesta, el cual constituye el formulario a ser utilizado para recolectar los datos, al realizarse en una forma rápida y cómoda.

En el caso del estudio planteado, la muestra estuvo conformada por aquellos sujetos que respondan el instrumento de recolección de información en el tiempo requerido y estimado para llevar a cabo el procesamiento respectivo. La elaboración del cuestionario al cual se ha hecho referencia esta conformada por 20 reactivos y es una prueba de actitud con una escala de Likert de 5 alternativas. En el se evaluó la opinión de los directivos de las pequeñas y medianas empresas acerca de sus competencias gerenciales, ello con relación a:

- Conocimientos

- Habilidades
- Rasgos y temperamento

- Motivación y necesidades

- Capacidad de adecuación o adaptación, generación y aplicación.

Puede agregarse que en general el cuerpo gerencial, en sus diferentes niveles, es el elemento responsable de los procesos administrativos, todas estas funciones como planificar, organizar, dirigir y controlar; son imprescindibles para el desarrollo de las labores de la organización, la gerencia es el factor, responsable de la organización como un todo (Antonorsi, 1999); de allí la importancia del gerente como un elemento clave llamado a dinamizar, a mover la organización.

Se entiende que la gerencia involucra grupos de personas, a veces muy diferentes que trabajan en conjunto y sincronizan sus actividades para lograr ciertos objetivos, producto de una gerencia efectiva en muchos casos y la implementación de determinadas capacidades. Otros autores, plantean en cuanto a los procesos de producción y distribución de una planta o en cualquier organización donde participan seres humanos, que han de adquirir para ello conocimientos, habilidades y formas de organización social (Gómez y Marqués, 1997).

La existencia de nuevos paradigmas en los cuales el conocimiento de la organización involucre a la gerencia bajo el precepto de que el gerente es un socio del proceso empresarial, en función de lo cual requiere atención a sus requerimientos de capacitación (Drucker, 2000); afirma la necesidad de involucrar la participación de una clara y evidente responsabilidad educacional y mas aun en la realidad gerencial en donde esta inmerso este individuo. 
Este manejo del conocimiento para optimizar el funcionamiento de las organizaciones en general constituye un aspecto de pertinencia social de las universidades frente a la realidad del empresariado, que se encuentra en el entorno, la cual muchas veces pasa desapercibida. Los programas de desarrollo gerencial en su mayoría vienen impuestos por convenios transnacionales o simplemente con alianzas estratégicas que no consideran las necesidades elementales de los gerentes de las pequeñas y medianas empresas, los cuales pueden ser presentados en estudios realizados por las instituciones de educación superior como parte de su vinculación con el sector productivo y atendiendo a sus propias necesidades.

\section{Retrospectiva de la Gerencia}

Desde los inicios del estudio de la variable dirección como objeto de análisis autores como Taylor y Fayol han planteado la necesidad de observar los procesos organizacionales con la finalidad de establecer mejoras en la cadena de producción. Ambos autores, a pesar de haber sido desarrollados en países diferentes (Estados Unidos de América y Francia) y sobre experiencias distintas; desde entonces argumentaron la necesidad de que las personas encargadas de dar dirección debían cumplir con algunos requisitos acordes a la realidad de entonces, como por ejemplo poseer experiencia en ese tipo de cambios plantea Viloria (2001).

Entre las teorías que han surgido en torno al estudio de los requisitos o competencias gerenciales, plantea Chia- venato (2003), fue desarrollado por los consultores de la firma Mckinsey y Asociados, el modelo de Thomas Peters, Robert Waterman, Tony Athos y Richard Pascale hacen énfasis en la interdependencia de siete elementos o variables organizacionales que se interrelacionan permanentemente entre ellas:

- Valores Compartidos (Shared Values)

- Estrategia (Strategy)

- Estructura (Structure)

- Personal (Staff)

- Sistemas (Systems)

- Estilo Gerencial (Style)

- Habilidades Organizacionales (Skills) El Modelo de las 7'S de McKinsey, considera entonces la importancia de realizar un análisis integral de la realidad organizacional, considerando todos los elementos que la conforman, destacándose entre ellos la importancia de establecer las habilidades organizacionales.

A lo largo de la historia, las diferentes teorías organizacionales, han debatido acerca de la existencia de diferentes estilos de gerenciar y para algunos fundamentalmente la gerencia representa un proceso racional y para otros técnico, y que en definitiva es una ciencia que se aprende mediante una formación adecuada y se perfecciona con el paso de los años, según Anzizu (1998).

En este sentido Anzizu (1998) plantea la necesidad de caracterizar al gerente bajo el llamado triangulo de la dirección, en el cual debe haber un equilibrio entre actitudes, conocimientos y capacidades así como las habilidades que deben ponerse en practica para ejecutar la dirección. Este autor hace mención a la 
Realidad de la formación gerencial en las pequeñas y medianas empresas Hernández, René; Silvestri, Karin; Añez, Silenis y Gamboa, Luisa

necesidad de establecer la necesidad de que las personas que están al frente de una empresa deben poseer una serie de habilidades y capacidades esenciales, y que las mismas debían estar relacionadas o bajo una visión compartida con los objetivos organizacionales.

En el ámbito global las nuevas tendencias gerenciales se enfocan a presentar las veloces e inéditas transformaciones que caracterizan a la sociedad actual según Drucker (1998) y Viloria (2001) las empresas deben desarrollar una capacidad de aprendizaje diferente que les permita asegurar su esencia, promover su crecimiento y crear su propio porvenir. Este aprendizaje debe inconsecuencia corresponderse con la realidad existente, con las demandas internas, en congruencia con las exigencias del entorno.

La formación gerencial requerida, debe corresponderse con una realidad que debe determinarse, coincidiendo ampliamente según lo planteado hasta ahora con la propuesta de Senge (2005) en la cual las organizaciones deben ser capaces de eliminar las resistencias al aprendizaje evidenciado a lo largo de su vida y desarrollado por las personas. Para el autor, las organizaciones que deseen aprender deben ejercitarse en cinco disciplinas integradas y complementarias:

- Desarrollo de la Maestría Personal: dirigido a entender mejor las características del entorno y la capacidad para aprehender los elementos y situaciones más relevantes del mismo que permitan promover una mejor respuesta a las realidades externas.

- Manejo de diferentes modelos mentales que posibiliten la adopción de nuevos enfoques, de maneras distin- tas de interpretar la realidad, desechando la tentación de anclarse en un solo estilo gerencial.

- Construcción de una visión compartida que se constituya en una fuerza energizante e inspiradora.

- Aprendizaje en equipo tendiente a potenciar las posibilidades de aprendizaje del personal dentro de un ambiente de confianza, complementación, crecimiento conjunto e intercambio de conceptos y experiencias, tanto dentro del propio equipo como de otros equipos dentro de la empresa.

- Pensamiento Sistémico, que permite identificar las interrelaciones de los agregados, los diferentes subsistemas o procesos considerando a la organización como un todo.

El planteamiento de Senge planteado realmente evidencia la urgencia de que debe analizarse la realidad de las organizaciones en forma continua, debe monitorearse en que circunstancias se encuentra la gerencia con la finalidad de establecer criterios idóneos tendientes a la formación gerencial, en correspondencia con las necesidades detectadas.

En Venezuela desde los años 80 se iniciaron estudios relacionados con la gerencia empresarial, desde entonces autores como Naim y Piñango (2000) plantearon en sus investigaciones, aproximaciones teóricas a la necesidad de generar lineamientos que estuviesen enmarcados en el entorno de las empresas venezolanas, es decir en su propia realidad.

A partir de allí se iniciaron estudios, recopilaciones y casos asociados con el día a día de las empresas venezolanas, orientados a plasmar su realidad cada vez más tendiente a mostrar las debilida- 
des y fortalezas que mostraron la llamada ilusión de armonía según Naim citado por Villalba (2000).

Los estudios llevados a cabo por Naim y Piñango (2000) evidenciaron la necesidad de abordar la realidad empresarial venezolana con la finalidad de generar teorías acordes con la detección de fortalezas y debilidades de su gerencia, es decir establecer investigaciones alrededor de una pertinencia local. Los estudios de estos autores generaron que un alto índice de empresarios no poseía formación profesional en el área de la gerencia, haciendo hincapié en el término de gerente empírico.

Para determinar la existencia de los gerentes empíricos, los cuales han obtenido el conocimiento en el área gerencial producto de la experiencia en el cargo, se realizaron investigaciones y diagnósticos por Naim y Piñango, que arrojaron estos resultados. El fortalecimiento de organizaciones como el Instituto de Estudios Superiores en Administración, tuvo su asidero y posicionamiento en el mercado empresarial venezolano, precisamente por determinar la realidad organizacional de las empresas venezolanas en un momento especifico y mantenerse monitoreando el entorno de las mismas, con la finalidad de planificar alternativas de capacitación acordes con sus exigencias.

Kliksberg (2002) plantea al respecto, la necesidad de establecer lineamientos claros en el manejo de la gerencia, sustentados en proporcionarle los insumos necesarios para manejarse con criterios de excelencia. Cabe considerar en cuanto al suministro de estos insumos a los que hace referencia el autor, los cuales se relacionan con una formación gerencial acordes con sus necesidades propias de capacitación, en forma localizada.

Hoy en día las empresas venezolanas en general enfrentan el reto de hacerse más competitivas en una economía globalizada, emprendiendo para ello costosos esfuerzos, para conocer y aplicar las más modernas y novedosas técnicas de gerencia. Independientemente del tamaño de las organizaciones, existen factores que inciden directamente en el desenvolvimiento de las actividades cotidianas, cuya direccionalidad parte de la gerencia.

En este escenario acontece la realidad de las pequeñas y medianas empresas venezolanas según se ha analizado hasta ahora en las teorías e investigaciones descritas, donde la aplicación de estrategias y manejo de la información inciden directamente en la toma de decisiones a corto, mediano y largo plazo, cuyo acierto dependerá de una serie de factores internos y externos; destacándose dentro de los que directamente puede manejar la organización la formación gerencial de los directivos.

Los continuos cambios en el ámbito político, social, cultural, económico y tecnológico del entorno, requieren que específicamente las pequeñas y medianas empresas, dada su permeabilidad ante el entorno identifiquen en forma constante las oportunidades y las amenazas, evalúen sus fortalezas y debilidades; en función de flexibilizar sus procedimientos y dar respuesta a las exigencias planteadas.

Es por ello, que Anzola (1995) señala la existencia de una serie de estos factores citados anteriormente, que inci- 
Realidad de la formación gerencial en las pequeñas y medianas empresas Hernández, René; Silvestri, Karin; Añez, Silenis y Gamboa, Luisa

den directamente y también son afectados por la empresa, entre ellos están: a) factores económicos; b) factores políticos y legales; c) factores sociales; d) factores tecnológicos y, e) factores educativos, entre otros.

Anzola (1995) hace referencia a que muchas veces, el éxito de una empresa depende del medio ambiente externo, en el cual esta se encuentra o relaciona. La demanda actual del entorno de flexibilidad y respuestas rápidas por parte de las organizaciones, exige en consecuencia una toma de decisiones fundamentada en una formación gerencial que le permita afrontar la dinámica y proporcionar directrices claras ante las exigencias generadas en forma continua.

La realidad de la Pequeña y Mediana Empresa debe atender a este requerimiento de formar profesionalmente al personal gerencial en función de la necesidad propia de capacitación en estos directivos, para enfrentar la orientación de los destinos de los distintos sectores de la actividad nacional y el manejo de los factores que le generan incertidumbre de manera: eficiente, oportuna y acertada. Este requerimiento de formar el llamado capital intelectual debe constituir un objetivo empresarial.

Autores como Bateman(2002) manifiestan que el éxito de una empresa se encuentra en la formación de su capital intelectual; se observa como en las empresas los activos fijos vienen siendo relegados; la capacidad de gestionar talento humano; la capacidad de gestionar el intelecto humano se está convirtiendo en la tendencia gerencial del presente, como consecuencia de lo anterior se ha desarrollado a nivel global un marcado interés por el capital intelectual, la creatividad, la innovación y el aprendizaje organizacional.

En tales circunstancias se hace necesario establecer algunas premisas acerca de factores intervinientes en las organizaciones dirigidas a la formación gerencial. Específicamente, el factor que marca esta diferencia es el capital intelectual. Para el efecto se señala a continuación una descripción de los elementos que conforman el capital intelectual según la óptica de Bateman (2000), quien hace dos grandes clasificaciones del capital intelectual así:

Capital Humano: que corresponde al conjunto de conocimientos, habilidades, actitudes, y destrezas de las personas que componen las organizaciones.

Capital estructural: conocimientos desarrollados y explícitos por las organizaciones.

Debe señalarse la importancia para la organización de establecer la importancia del Capital Intelectual, en cuanto a lo concerniente a los activos humanos (educación, formación profesional, conocimientos específicos del trabajo, habilidades) referidos por Edvinsson.

Se plantea así la necesidad de determinar la realidad de la formación gerencial de las pequeñas y medianas empresas lo cual involucra medir una serie de competencias existentes y hacer una detección de requerimientos específicos, para ello debe entonces caracterizarse como es y que requiere un gerente de una pequeña y mediana empresa venezolana.

Anzola (1995) hace un esbozo acerca de la existencia de una serie de características predominantes de la pe- 
queña y mediana empresa y entre ellas destaca:

"El mercado local o regional es el objetivo predominante de la pequeña y mediana empresa. Esta característica depende de la habilidad del empresario para ofrecer un producto o servicio excelente o de mejor calidad en un mercado particular y así definirlo en términos geográficos, enfocado al mercado local." (p.5).

Según este autor las habilidades forman parte de los requerimientos organizacionales para sobrevivir ante un ambiente de incertidumbre, se plantea entonces el problema de establecer cuales de ellas son requeridas para desempeñar cargos gerenciales, considerando además que en función de estas habilidades se encuentra el futuro de la organización.

Específicamente se amerita conocer, cuales son las habilidades, conocimientos y características personales de los directivos que conforman la realidad de la formación gerencial en las Pequeñas y Medianas Empresas (PyME), las cuales constituyen uno de los pilares fundamentales de la economía venezolana, estimándose según Palacios (1999: 21) en más del $65 \%$ de las compañías existentes en el país.

El análisis procedente de lo planteado hasta ahora, refiere la necesidad de realizar un estudio exhaustivo, es decir determinar las características de la gerencia de las Pequeñas y Medianas Empresas, que pueda proporcionar información y el conocimiento necesario acorde con las necesidades actuales, de este sector empresarial en Venezuela.

Al respecto Palacios (1999: 285) refiere la existencia de competencias fundamentales que deben ser desarro- lladas, para cumplir con éxito el rol de emprendedor, destacando la necesidad del conocimiento técnico, auto confianza, la capacidad de poner un esfuerzo adicional, identificarse con el negocio, tener iniciativa, saber innovar, ser capaz de conseguir información, tener olfato para los negocios, orientación al cliente y orientación al logro.

Para establecer la existencia de estas competencias necesariamente según lo planteado hasta ahora, debe realizarse un análisis de la realidad gerencial para observar en que medida se encuentra identificada con el planteamiento de $\mathrm{Pa}$ lacios, considerando la detección de necesidades previa citadas por el autor.

Por otro lado, Anzizu (1999: 64) refiere la necesidad de fomentar la capacidad técnica como esencial, la cual debe tener la persona que este al frente de una empresa, compartiendo así la necesidad e importancia de la capacitación de la gerencia, para la obtención de resultados exitosos.

No contrasta esta información con el estudio realizado por la misma autora posteriormente, en el cual obtuvo entre los gerentes encuestados en su investigación, sujetos de información de la gerencia de alto nivel que han tenido múltiples oportunidades para recibir una excelente educación, que están muy bien informados sobre las tendencias y modelos mas modernos a nivel gerencial, no implicando según se pudo obtener, que su conducta sea congruente con los objetivos organizacionales según Granell (2000: 115).

Esta información obtenida de los estudios de Granell indica el requerimiento de planificar una formación gerencial en función de las necesidades personales y organizacionales, si los resultados 
Realidad de la formación gerencial en las pequeñas y medianas empresas Hernández, René; Silvestri, Karin; Añez, Silenis y Gamboa, Luisa

que pretenden obtenerse van a ir en beneficio no solo de la organización sino también del individuo bajo una concepción sistémica; tomando en cuenta para ello el establecimiento de un perfil de competencias y una detección de necesidades de capacitación.

En función de lo descrito Drucker (2000) plantea la existencia de nuevos paradigmas en los cuales el conocimiento de la organización debe involucrar a la gerencia bajo el precepto de que el gerente es un socio del proceso empresarial, en función de lo cual requiere atención a sus requerimientos de capacitación; esta afirmación amerita la participación de una clara y evidente responsabilidad educacional, de la realidad gerencial en donde esta inmerso este individuo.

\section{Las Pequeñas y Medianas Empresas en Venezuela}

En Venezuela existen diversas formas de establecer el tamaño de una empresa, la más generalizada se fundamenta en identificar el número de trabajadores, aunque también se puede considerar el monto del capital o de los activos. El organismo del Estado venezolano para el manejo y administración de la información referente a las empresas, es la Oficina Central de Estadística e Informática (OCEI), el criterio utilizado en forma explicita por esta dependencia para la clasificación de las empresas esta en función del número de empleados.

\section{La Formación Profesional}

Autores como Torres (2002) definen a la formación profesional como el conjunto de políticas, estrategias y procesos que están orientados a formar y capacitar de manera continua y permanente a la fuerza laboral. La definición de formación profesional según su objetivo esta contemplado refiere el citado autor, en el punto 2.1 de la recomendación 150 de la Organización Internacional del Trabajo (OIT) como:

"El de descubrir y desarrollar las aptitudes humanas para una vida activa, productiva y satisfactoria y, en unión de las diferentes formas de educación, mejorar las aptitudes individuales para comprender individual y colectivamente cuanto concierne a las condiciones de trabajo y el medio social e influir en ellos" (p. 25).

El autor al cual se ha hecho referencia, establece que la formación profesional es un derecho reconocido en la normativa laboral, de carácter internacional y como tal esta condicionado al goce del derecho al trabajo, y al mismo tiempo vinculado al ejercicio del derecho a la educación, la cual se configura con una dimensión específica.

Según Chiavenato (2000) la formación profesional comprende una etapa del individuo en la cual se le prepara para ejercer en un determinado mercado de trabajo, es decir comprende la educación profesional, institucionalizada, Ilamada también nivel educativo, en el cual se proporcionan al individuo una serie de conocimientos para una futura profesión. Sus objetivos son amplios y a largo plazo, puede impartirse en escuelas, y también en las propias empresas.

La formación profesional en Venezuela esta consagrada en la Constitución de la Republica Bolivariana de Venezuela 
cuando en el artículo 79 especifica en función de los jóvenes y las jóvenes el derecho de ser sujetos activos del proceso de desarrollo. Esta clara delimitación permite desarrollar el capital intelectual a través de la normativa favoreciendo así al fortalecimiento del capital humano, estimulando la participación del gobierno en fomentar programas direccionales orientados básicamente a la capacitación.

\section{Resultados obtenidos en la Investigación}

La interpretación y análisis de los resultados obtenidos en la investigación, se fundamentaron básicamente en algunos aspectos especíicos, los cuales son:

- Nivel en el cual fue medida la variable Formación Gerencial.

- Alcance de los objetivos de la investigación.

- Pertinencia del estudio en función del entorno al cual se encuentran circunscritas las pequeñas y medianas empresas (PyME) en los municipios $\mathrm{Ca}$ bimas y Lagunillas del estado Zulia.

En el análisis también se indica si el estudio respondió a los objetivos planteados y en cuanto a la discusión de los resultados se considera como el aspecto más importante que se va a tener en cuenta en toda la investigación; en virtud de permitir el contraste de la teoría y la praxis (Bernal, 2000).

La realización del procesamiento de los datos obtenidos mediante la aplicación de los instrumentos de recolección de información, estuvo orientada a determinar la realidad de la formación gerencial de los directivos en las Pequeñas y Medianas Empresas (PyME) en la muestra seleccionada, asistido por la aplicación de la Estadística Descriptiva para la variable de estudio. El análisis procedente de la información recolectada en cuanto a las necesidades de formación puede abordarse al plantear que la formación gerencial consiste en el proceso gradual y sistemático de mejora de los conocimientos, destrezas, actitudes y rendimientos o resultados alcanzados en aquellos miembros de una organización, sobre los cuales recaen las responsabilidades de dirección.

A efectos de llevar a cabo la interpretación de los resultados obtenidos se consideró el análisis estadístico, de la variable Formación Gerencial. En cuanto a los datos recolectados por el cuestionario Tipo A, el cual mide las Competencias Gerenciales, se utilizaron las técnicas denominadas medidas de tendencia central, a través del uso de la media o promedio aritmético $(X)$, y para la variabilidad se aplicó la desviación estándar.

La utilización de la media es útil y precisa según (Sierra, 2001), tomando como referencia la categorización de ítemes, indicadores, dimensiones así como la variable en estudio. Sobre este fundamento y para facilitar la interpretación, se diseñó un cuadro de rango, intervalo y categoría. El rango de la aplicación comprende valores que representan determinadas categorias, en función de las cuales se asignaron ciertos parámetros.

En forma puntual se observó que los rangos contemplaban la escala desde el 1 hasta el 5, donde cada intervalo correspondiente esta determinado por una secuencia de 1,80 denominándose categoría de análisis para la interpretación del promedio, iniciándose desde el 1 hasta el 
Realidad de la formación gerencial en las pequeñas y medianas empresas Hernández, René; Silvestri, Karin; Añez, Silenis y Gamboa, Luisa

5; y las categorías comprenden desde las de muy bajo nivel hasta las de muy alto nivel de incidencia. Ver Tabla 1.

La medida de variabilidad índica la dispersión de los datos en la escala de medición. En la investigación realizada se empleo la desviación estándar, para indicar el grado de dispersión de las respuestas dadas por los gerentes encuestados, con relación a la escala de medición utilizada y su rango respectivo. Se entiende entonces que la desviación estándar es el promedio de dispersión de las puntuaciones con respecto a la media.

El rango, también llamado recorrido representa la diferencia entre las puntuaciones mayores y menores obtenidas, es decir, la mayor es seis (6) y la mínima es cero (0) respectivamente, las medidas de la variabilidad más utilizadas comúnmente según (Hernández y col., 2000) son el rango, la desviación estándar y la varianza.

De las medidas de variabilidad citadas, fueron calculadas para la elaboración del cuadro que se presenta a continuación el rango, el intervalo y la categoría para ubicar el análisis de la desviación estándar. Ver Tabla 2.

Con relación a la interpretación de los resultados, se elaboró una serie de cuadros ilustrativos para la variable, dimensiones e indicadores, los cuales representan las medidas utilizadas para efectos de generar análisis cónsonos con sus respectivos valores arrojados a través del procesamiento de datos.

Tabla 1

Categoría de Análisis para la Interpretación del Promedio

\begin{tabular}{ccccc}
\hline Rango & \multicolumn{3}{c}{ Intervalo } & Categoría \\
\hline 1 & 4,21 & - & 5 & Muy Alto Nivel \\
2 & 3,41 & - & 4,20 & Alto Nivel \\
3 & 2,61 & - & 3,40 & Moderado Nivel \\
4 & 1,81 & - & 2,60 & Bajo Nivel \\
5 & 1 & - & 1,80 & Muy Bajo Nivel \\
\hline
\end{tabular}

Fuente: Elaboración propia con base a las encuestas (2007).

Tabla 2 Categoría de Análisis para la Desviación Estándar

\begin{tabular}{ccccc}
\hline Rango & \multicolumn{3}{c}{ Intervalo } & Categoría \\
\hline 1 & 4,81 & - & 6 & Muy Alta Dispersión \\
2 & 3,61 & - & 4,80 & Alta Dispersión \\
3 & 2,41 & - & 3,60 & Moderada Dispersión \\
4 & 1,21 & - & 2,40 & Baja Dispersión \\
5 & 0 & - & 1,20 & Muy Baja Dispersión \\
\hline
\end{tabular}

Fuente: Elaboración propia con base a las encuestas (2007). 
El análisis de la variable de estudio Formación Gerencial, en cuanto a la dimensión competencias gerenciales tiene su fundamentación en el planteamiento de (Hernández, 2002) referido a la existencia de competencias laborales las cuales se definen como la capacidad productiva de un individuo que se mide en términos de desempeño atendiendo a un determinado contexto laboral y esta fundamentado en los conocimientos, habilidades, destrezas y actitudes necesarias para la realización de un trabajo efectivo y de calidad.

El autor (Benavides, 2002) agrega además la necesidad de considerar dentro de la clasificación de competencias la existencia de las competencias laborales o gerenciales. Al mencionarse a estas competencias se hace referencia a que dentro de ellas se contemplan los conocimientos, habilidades, auto concepto, rasgos y temperamento, motivación y necesidades propias de los individuos que ejercen la gerencia.

En este orden de ideas (Hernández, 2002) plantea la existencia de dos tipos de competencias que provocan un patrón y norma para la selección de personal, la planeación, la sucesión y la evaluación de la actuación para el desarrollo de la persona en una organización, estas son:

- Las competencias diferenciadoras. Se definen como las que distinguen a un trabajador con una actuación superior a los de una actuación media.

- Las competencias umbral. Definidas como las que se necesitan para lograr una actuación media o minimamente adecuada.
Las competencias, según este autor pueden consistir en:

- Motivos. La necesidad de logros.

- Rasgos de carácter. Confianza en sí mismo, autocontrol, resistencia al estrés.

- Concepto de sí mismo. Actitudes y valores.

- Contenido de conocimientos técnicos interpersonales. El contenido de conocimientos rara vez sirve para diferenciar a los trabajadores de actuación superior de los que tienen una actuación media.

- Capacidades cognoscitivas y de conducta. Pueden ser ocultas (razonamiento deductivo e inductivo) y observables (capacidad de escuchar e interactuar). Las competencias sirven para predecir conductas que, a su vez, predicen el desempeño concreto.

En relación con el primer objetivo específico diseñado en la investigación elaborado en función de establecer el perfil de competencias gerenciales de los directivos de las PyME venezolanas los resultados obtenidos luego de la aplicación del instrumento en los gerentes de las PyME en la COL, denominados sujetos de información, evidenciaron un promedio de 4,76 para la dimensión competencias gerenciales con una categoría de muy alto nivel, que indica el uso de competencias gerenciales en un mayor porcentaje, con una desviación de 0,49 lo cual hace inferir una muy baja dispersión en las respuestas. Ver Tabla 3.

Efectivamente los elementos citados por Benavides (2001) fueron los que se consideraron para conformar los indicadores de la dimensión competencias gerenciales en esta investigación, com- 
Realidad de la formación gerencial en las pequeñas y medianas empresas Hernández, René; Silvestri, Karin; Añez, Silenis y Gamboa, Luisa

Tabla 3

Variable: Formación Gerencial

\begin{tabular}{ccc}
\hline Dimensión & Promedio & Desviación \\
\hline Competencias Gerenciales & 4,76 & 0,49 \\
Promedio General & 4,76 & 0,49 \\
\hline
\end{tabular}

Fuente: Elaboración propia con base a las encuestas (2007).

prendidos por los conocimientos, habilidades, rasgos y temperamento, motivación y necesidades, capacidad de adecuación, generación y aplicación.

Según estas puntuaciones resultantes, dentro de las competencias gerenciales aportadas por las unidades de información, se infiere que se consideran con mayor importancia y gran significado para el desempeño de la gerencia la capacidad de adecuación, generación y aplicación con un promedio de 4,99 ubicándose como de muy alto nivel y con una desviación de 0,04 tipificada como de muy baja dispersión.

El mismo análisis se puede realizar con la competencia que designaron contrariamente con menor importancia, la cual esta representada por los conocimientos, la cual esta ubicada con un valor de 4,14 considerada como de alto nivel pero que presentó una desviación de 1,23 reflejando una baja dispersión en las respuestas obtenidas. Ver Tabla 4.

Según estas cifras puede inferirse, entre los resultados obtenidos en torno al indicador conocimientos, que efectivamente no había consenso en relación con esta competencia, ni tampoco un total dominio por parte de los consultados, probablemente debido a conocimientos insuficientes a aplicar en el campo gerencial, obtenidos mayormente por experiencia adquirida en el ejercicio del cargo de- sempeñado, más que en el curso de una carrera gerencial.

Al analizar específicamente el indicador relacionado con los conocimientos en el área gerencial, el promedio general obtenido fue 4,76 , considerado como de muy alto nivel y la desviación correspondiente es de 0,49 categorizada como de muy baja dispersión. De los ítemes que conforman este indicador, se obtuvo que el mayor promedio coincidiera en definir a los conocimientos gerenciales, como producto de la experiencia en el ejercicio del cargo, mientras que la desviación más elevada giró en torno al ítem 1 donde se vinculaba a los conocimientos con el curso de una carrera universitaria (Ver Tabla 5).

La información obtenida en la práctica es sustentada teóricamente por autores como (Werther y Davis, 2000) quienes plantean que en las empresas además de la información en general acerca de la planeación de una carrera profesional también es necesario proporcionar información específica, de carácter formativo, que puede describirse como capacitación y desarrollo respecto a la organización misma.

Dentro de este marco (Blanchard, 1997) expone que debe hablarse en serio de la capacitación ejecutiva o formación gerencial, para el manejo de las pequeñas empresas; es decir, debe hacerse 
Tabla 4

Dimensión: Competencias Gerenciales

\begin{tabular}{lcc}
\hline Indicadores & Promedio & Desviación \\
\hline Conocimientos & 4,14 & 1,23 \\
Habilidades & 4,97 & 0,21 \\
Rasgos y Temperamento & 4,75 & 0,70 \\
Motivación y Necesidades & 4,95 & 0,25 \\
Capacidad de Adecuación, Generación y Aplicación & 4,99 & 0,04 \\
Promedio General & 4,76 & 0,49 \\
\hline
\end{tabular}

Fuente: Elaboración propia con base a las encuestas (2007).

Tabla 5

Indicador: Conocimientos

\begin{tabular}{ccc}
\hline Ítemes & Promedio & $\begin{array}{c}\text { Desviació } \\
\mathrm{n}\end{array}$ \\
\hline 1 & 3,26 & 2,01 \\
2 & 4,49 & 0,88 \\
3 & 4,67 & 0,81 \\
Promedio General & 4,76 & 0,49 \\
\hline
\end{tabular}

Fuente: Elaboración propia con base a las encuestas (2007).

participe a los gerentes de las organizaciones, para lograr la transferencia de aprendizaje y ello solo es posible cuando el proceso de fijación de conocimientos, se planifica considerando el entorno en el cual se encuentra la organización y atendiendo a las necesidades reales de la gerencia, desestimando para ello a las modas del mercado de la capacitación.

En relación con las habilidades, como indicador se obtuvo que el promedio de las respuestas se orientará mayormente a la práctica de las habilidades técnicas, con un valor de 5 considerado como el valor más alto de una categoría de muy alto nivel y una desviación de 0 ubicándose como de muy baja dispersión.
Las otras dos habilidades consideradas en menor proporción por las unidades de información, pero con un promedio igualmente significativo son las habilidades de trato con las personas y las habilidades administrativas donde ambas obtuvieron una desviación de 0,32 indicando una muy baja dispersión en las respuestas. Ver Tabla 6.

Al analizar los resultados vinculados con el indicador de rasgos y temperamento, el promedio mayor es de 4,97 considerado como de muy alto nivel, obtenido por el ítem que plantea la existencia de características personales especiales en los gerentes. La desviación estándar más elevada es de 1,12 tipificada como de muy baja dispersión, representada en el ítem referido a la aplicación de un temperamento flexible. Este resultado, refleja la existencia de ciertos paradigmas asociados con el uso de temperamentos recios en la organización, para la obtención de una mayor productividad por parte del gerente hacia los subordinados.

El temperamento se asocia con el estilo para gerenciar y el mismo responde a una característica de personalidad propio y característico del individuo según 
Realidad de la formación gerencial en las pequeñas y medianas empresas Hernández, René; Silvestri, Karin; Añez, Silenis y Gamboa, Luisa

Tabla 6 Indicador: Habilidades

\begin{tabular}{ccc}
\hline Ítemes & Promedio & Desviación \\
\hline 4 & 5 & 0 \\
5 & 4,95 & 0,32 \\
6 & 4,95 & 0,32 \\
Promedio General & 4,97 & 0,21 \\
\hline
\end{tabular}

Fuente: Elaboración propia con base a las encuestas (2007).

(Thorne, 1993) y (Bateman y col., 2000), dado que la concentración de respuestas en torno a las características personales especiales reflejan el propio sentido de los encuestados de considerar como requisito ciertos estereotipos como la edad, sexo, entre otros. Ver Tabla 7.

Los datos obtenidos de la aplicación del instrumento en cuanto al indicador motivación y necesidades, arrojó un promedio de respuestas de 4,95 valor considerado como de muy alto nivel, es decir la mayoría coincidió en afirmar la importancia de esta competencia, y lo significativo de estar motivado y alineado con los objetivos organizacionales, para el ejercicio de la práctica gerencial.

En menor grado con un valor de 4,92 , se ubicaron las respuestas acerca de vincular a la motivación con el ejercicio de la gerencia y la obtención de eficiencia correspondientes a los ítemes 11 y 12 .

El promedio general de la desviación fue mínima de un 0,25 establecida como de muy baja dispersión, evidenciando así la concentración de las respuestas, en torno al requerimiento de la necesidad de alinear la motivación de los gerentes con los objetivos organizacionales. El más alto valor lo obtuvieron los ítemes referidos a la existencia de un clima
Tabla 7 Indicador: Rasgos y Temperamento

\begin{tabular}{ccc}
\hline Ítemes & Promedio & Desviación \\
\hline 7 & 4,59 & 1,04 \\
8 & 4,51 & 1,12 \\
9 & 4,92 & 0,48 \\
10 & 4,97 & 0,16 \\
Promedio General & 4,75 & 0,70 \\
\hline
\end{tabular}

Fuente: Elaboración propia con base a las encuestas (2007).

de trabajo, que permite la satisfacción de las necesidades de afiliación y necesidades económicas. Contrariamente la mayor dispersión se centro en no considerar necesarios estar motivado para el ejercicio de la gerencia y la obtención de eficiencia. Ver Tabla 8.

De los seis (6) ítemes que midieron este indicador, el total de ellos obtuvo promedios considerados como de muy alto nivel; es decir marcan la existencia de una aceptación generalizada de los gerentes, en la importancia de la satisfacción de las necesidades y en la motivación como factores de interés para incrementar el potencial. Ese mismo resultado se obtuvo en las desviaciones valores, promedios de 0,25 demostrando que la mayoría de las unidades de información considera importantes estos indicadores con categoría como de muy baja dispersión.

Finalmente los resultados para el instrumento Tipo A procesado, demostraron respecto a la capacidad de adecuación, generación y aplicación un promedio orientado hacia un valor de 4,99 considerado como de muy alto nivel, mientras que la desviación estándar fue de 0,04 es decir de muy baja dispersión. 


\begin{tabular}{ccc}
\multicolumn{3}{c}{$\begin{array}{c}\text { Tabla } 8 \\
\text { Indicador: Motivación } \\
\text { y necesidades }\end{array}$} \\
\hline Ítemes & Promedio & Desviación \\
\hline 11 & 4,92 & 0,35 \\
12 & 4,92 & 0,35 \\
13 & 4,95 & 0,22 \\
14 & 4,95 & 0,22 \\
15 & 4,97 & 0,16 \\
16 & 4,97 & 0,16 \\
Promedio General & 4,95 & 0,25 \\
\hline
\end{tabular}

Fuente: Elaboración propia con base a las encuestas (2007).

Estos valores indican, al comparar los ítemes del cuestionario que en mayor proporción el común de los gerentes consideró importante, para el ejercicio de su actividad directiva, poner en práctica las capacidades para adecuarse o adaptarse, la capacidad para generar respuestas acordes con las exigencias de la situación a presentarse, la capacidad de aplicación de nuevas técnicas y adaptarse fácilmente a las condiciones que varían constantemente.

En cuanto a los ítemes diseñados para medir el indicador capacidad de adecuación, se obtuvo que la dispersión fue de 0 considerada como de muy baja dispersión, es decir se presenta un consenso en torno a las respuestas de las unidades de información al estimar como importante esta capacidad para el desempeño de la gerencia.

Solo un 0,16 de la puntuación de las unidades de información se orientó a considerar la capacidad de aplicación de nueva técnicas, políticas y estrategias como significativa. Ver Tabla 9.
Tabla 9

Indicador: Capacidad de adecuación y aplicación

\begin{tabular}{ccc}
\hline Ítemes & Promedio & Desviación \\
\hline 17 & 5 & 0 \\
18 & 5 & 0 \\
19 & 4,97 & 0,16 \\
20 & 5 & 0 \\
Promedio General & 4,99 & 0,04 \\
\hline
\end{tabular}

Fuente: Elaboración propia con base a las encuestas (2007).

Esta continua búsqueda de alinear los esfuerzos organizacionales con el alcance de los objetivos ha hecho posible la participación de gerentes de las PyME en la investigación realizada cuyo objetivo medular estaba constituido precisamente en determinar la realidad de la formación gerencial de los directivos de las pequeñas y medianas empresas en los municipios $\mathrm{Ca}$ bimas y Lagunillas del estado Zulia.

\section{Conclusiones}

En el contexto explorado y atendiendo a los resultados de la investigación a continuación se presentan las conclusiones derivadas del proceso de análisis:

En atención al primer objetivo especifico referido a establecer el perfil de competencias gerenciales de los directivos de las PyME venezolanas se llegó a la conclusión de que el mismo esta conformado por una serie de indicadores como los conocimientos, habilidades, rasgos y temperamento motivación y necesidades, capacidad de adecuación, generación y aplicación. 
Realidad de la formación gerencial en las pequeñas y medianas empresas Hernández, René; Silvestri, Karin; Añez, Silenis y Gamboa, Luisa

En torno al indicador conocimientos las respuestas arrojaron que los mismos habían sido obtenidos en un mayor porcentaje como producto de cursar una carrera universitaria.

Dentro del indicador habilidades se obtuvo que la totalidad de la muestra coincidiera en responder que las habilidades técnicas son necesarias para el ejercicio de la gerencia.

De acuerdo a los resultados para el indicador rasgos y temperamento se concluye que el mayor porcentaje de los encuestados estiman que los gerentes tienen características personales especiales.

Respecto al indicador motivación y necesidades se concluye que los gerentes consideran importante la satisfacción de las necesidades de afiliación y las de tipo económica.

El indicador considerado con un mayor porcentaje de respuestas es la capacidad de adecuación y aplicación, por lo que se concluye que es considerado de gran importancia para quienes ejercen la gerencia de las PyME.

En cuanto al objetivo especifico trazado para determinar la Formación Profesional de los gerentes de las PyME, se concluye en torno al indicador nivel educativo que el mayor porcentaje de los consultados manifestó en un 58,97 \% poseer un nivel de educación universitario.

Con relación al indicador capital intelectual se obtuvo que la participación de las empresas en la formación profesional del personal se limita mayormente a promover la capacitación del personal gerencial y que la capacitación del capital intelectual para un alto porcentaje constituye una prioridad, específicamente para el personal gerencial.
La capacitación del capital intelectual según los datos aportados demuestra que en las PyME se implementan con mayor incidencia los talleres como estrategia para facilitar el aprendizaje organizacional.

\section{Consideraciones Finales}

Dentro de las recomendaciones que pueden plantearse a los directivos de las pequeñas y medianas empresas venezolanas se encuentran básicamente las siguientes:

- Debe considerarse una apertura al conocimiento en el área gerencial, estableciendo alianzas que les permitan obtener la posibilidad de capacitar al personal de la organización en una mayor proporción a la actual.

- La gerencia dentro de su responsabilidad de mantenerse actualizada para hacer frente a los cambios del entorno, debe realizar un auto-análisis que determine realmente hacia donde quiere llegar el directivo y en que medida esta dispuesto a formar parte de una verdadera formación gerencial, alineada con los objetivos organizacionales.

\section{Referencias Bibliográficas}

Antonorsi, M. (1999). Guía práctica de la empresa competitiva. Caracas, Venezuela: Edición de Venezuela Competitiva. Universidad Metropolitana.

Anzizu, J. (1998). El Management ¿Arte o Ciencia? Madrid, España: Fondo Editorial Español.

Anzola, G. (1995). Las Pequeñas y Medianas Empresas, un análisis inmediato. Editorial Trillas. México DF. 
Armas, V. (1999). ¿Qué necesitan las Pequeñas Empresas? Revista Debates IESA. Volumen 5 No.1. Caracas, Venezuela.

Bateman, T. y Snell, S. (2000). Administración una ventaja competitiva. México. D.F: Editorial Mc. Graw Hill.

Chiavenato, I. (2000). Administración de Recursos Humanos. Santa Fé de Bogota: Editorial Mc Graw Hill.

Drucker, P. (1998). EI Nuevo Cambio de la Productividad. Harvard Business Review. Noviembre-Diciembre de 1995.

Drucker, P. (2000). Las Nuevas Realidades. Bogotá, Colombia: Editorial Norma, Santa Fe de Bogotá.

Granell, E. (1994). Recursos Humanos y Competitividad en Organizaciones Venezolanas. Caracas, Venezuela: Ediciones IESA, pp.116.

Granell, E.; Garaway, D. y Malpica, C. (2000). Éxito gerencial y cultura. Caracas, Venezuela: Ediciones IESA. pp.192.

Kliksberg, B. (2002). Hacia una economía con rostro humano. Universidad del Zulia (LUZ), Maracaibo.
Naim, M. y Piñango, R. (2000). Venezuela: Una Ilusión de Armonía. Caracas Venezuela: Editorial IESA.

OCEI. Informe sobre Desarrollo Humano en Venezuela, 2000. CDB Publicaciones, 2001. Venezuela.

Palacios A., Luis E. (1999). Sabiduría popular en la empresa venezolana. Caracas: BBV-Banco Provincial: Espiñeira, Sheldon y Asociados; Universidad Católica Andrés Bello. 316 p.

Reza, J. (1995) Como Diagnosticar las necesidades de capacitación en las organizaciones. México, DF: Editorial Panorama.

Senge, Peter (2005). La Quinta Disciplina. Buenos Aires, Argentina: Editorial GRAMICA.

Torres, S. (2002). Competencias Laborales. Caracas, Venezuela: Editorial Texto, C.A.

Villalba, J. (2000). Las Empresas Venezolanas. Caracas Venezuela: Editorial IESA.

Viloria, E. (2001). ¿Qué es una Organización? Caracas, Venezuela: Editorial PANAPO. 